\title{
Análisis de las Curvas de Gelatinización de Almidones Nativos de tres Especies de Ñame: Criollo (Dioscorea alata), Espino (Dioscorea rotundata) y Diamante 22
}

\author{
Rodrigo D. Salgado-Ordosgoitia(1)*, Ana L. Paternina-Contreras ${ }^{(2)}$, Carlos S. Cohen-Manrique ${ }^{(1)}$, \\ Jhonatan A. Rodríguez-Manrique ${ }^{(1)}$ \\ (1) Facultad de Ciencias Básicas, Ingeniería y Arquitectura. Corporación Universitaria del Caribe CECAR, Sincelejo, \\ Colombia. (e-mail: rodrigo.salgado@cecar.edu.co) \\ (2) Universidad de Sucre, Departamento de Ingeniería Agroindustrial, Procesos Agroindustriales y Desarrollo Sostenible, \\ Sincelejo - Sucre - Colombia.
}

* Autor a quien debe ser dirigida la correspondencia

Recibido Ago. 24, 2018; Aceptado Nov. 20, 2018; Versión final Ene. 20, 2019, Publicado Ago. 2019

\begin{abstract}
Resumen
El objetivo del presente estudio fue analizar las curvas de gelatinización de almidones nativos, extraídos de las especies de ñame Criollo, Espino y Diamante 22. Metodológicamente, se utilizó un reómetro y un analizador rápido de almidón. Se concluye que la temperatura inicial de gelatinización fue mayor para las especies Criollo y Diamante 22. La mayor viscosidad se obtuvo en la especie Espino. Las especies Criollo y Diamante 22 presentaron mayor estabilidad y menor tendencia a la retro-degradación potencializando su uso en alimentos que requieran mantener un gel de consistencia estable a procesos de cocción y en productos que necesiten enfriamiento durante su preparación. Esto sugiere su posible uso en formulaciones de alimentos que requieran el rápido desarrollo de la viscosidad y un gel de consistencia estable.
\end{abstract}

Palabras clave: tubérculo; amilosa; amilopectina; viscosidad

\section{Analysis of the Gelatinization Curves of Native Starches of three Species of Yam: Criollo (Dioscorea alata), Hawthorn (Dioscorea rotundata) and Diamond 22}

\begin{abstract}
The objective of the present study was to analyze the gelatinization curves of native starches extracted from the Criollo, Espino and Diamante 22 yam species. Methodologically, a rheometer and a rapid starch analyzer were used. It was found that the initial gelatinization temperature was higher for the Criollo and Diamante 22 species. The highest viscosity was obtained in the Hawthorn species. Finally, the Criollo and Diamante 22 species presented greater stability and less tendency to retro degradation favoring their use in foods that require to maintain a gel of stable consistency to cooking processes and in products that need cooling during their preparation. This suggests their possible use in food formulations that require fast development of viscosity and a gel of stable consistency.
\end{abstract}

Keywords: tuber; amylose; amylopectin; viscosity 


\section{INTRODUCCIÓN}

En Colombia, principalmente en el departamento de Sucre, la agricultura es una de las principales actividades económicas que comúnmente practica la población. Esta actividad es considerada como una alternativa de sustento económico e inclusive de sostenimiento alimentario para las diferentes zonas rurales y urbanas de la localidad. Entre los principales productos emanados de dicha actividad, es posible destacar el predominio e importancia que tiene para los cultivadores locales un producto amiláceo como el ñame, ya que este tubérculo cuenta con demanda inmutable en el mercado, debido a que tradicionalmente se consume posterior a la cocción a vapor, en platos autóctonos de la región como los denominados "sancochos" y comúnmente es empleado en la industria de la repostería para la elaboración de dulces y postres. La planta de ñame (Reino: Plantae, División: Magnoliophyta, Clase: Liliopsida, Orden: Dioscoreales, Familia: Dioscoreaceae, Género: Dioscorea) puede ser definida como una planta enredadera trepadora con tallos que pueden alcanzar hasta más de tres metros de altura, se caracteriza porque su parte alimenticia es la raíz, la cual, es llamada tubérculo y es muy apetecida por sus valores nutricionales y aspectos de sabor. La planta de ñame contiene en su raíz grandes cantidades de almidón, por lo que se considera una fuente transcendental de energía (González Vega, 2012). En cuanto a su producción, existen registros obtenidos por el Departamento Administrativo Nacional de Estadística (DANE) de Colombia, provenientes de estudios agroforestales que analizaron el periodo comprendido entre los años 1987 y 2016, que indican que el departamento de Sucre es uno de los principales productores de ñame en el país, ocupando el cuarto lugar para el último periodo en mención. Es por ello que este tubérculo ocupa el tercer lugar en cuanto volúmenes de producción en comparación con los cultivos cosechados en el departamento en el año 2016, evidenciándose que dicha producción solo es superada por la producción de arroz secano mecanizado y por la producción de yuca que ocupan el primer y segundo lugar respectivamente, pero superando casi al doble, la producción de maíz tecnificado que es una de las principales actividades económicas en la agricultura en algunos departamentos de la costa caribe colombiana (DANE, 2016).

Sin embargo, a pesar de la alta cantidad producida en la región, la agrocadena de este tubérculo no ha sido considerado como un producto de primera necesidad para contribuir en la solución de problemas de seguridad alimentaria de la población y como consecuencia de este panorama, aún el desarrollo de las actividades productivas de este cultivo se realiza de forma empírica, careciendo de herramientas de desarrollo tecnológico que ayuden al mejoramiento de los resultados productivos e incremente aspectos competitivos. Sumado a esto, se resalta también la carencia de desarrollo para la agrocadena en los siguientes eslabones productivos, por lo cual, existen actualmente bajos niveles de transformación de esta materia prima, lo que no solo limita la generación de valor agregado para este producto sino que también restringe la generación de ventajas competitivas por la obtención de productos diferenciados para los participantes del sector, lo cual sin duda alguna requiere alternativas de procesamiento e intervención que amplíen el portafolio de uso y aprovechamiento del ñame para la elaboración de productos con mayor grado de madurez tecnológica que represente un impulso para esta agrocadena y para los participantes involucrados en ella (Alvis et al., 2016).

Así mismo, un panorama similar al del departamento de Sucre se encuentra en el ámbito nacional, donde el ñame no está considerado como producto prioritario, lo cual intensifica la problemática que enfrenta esta agrocadena e incrementa el desinterés de los empresarios involucrados de este sector a nivel local. Sin embargo, a nivel internacional principalmente en las regiones tropicales, el ñame es una de las fuentes alimenticias primordiales de la población, sobre todo en los continentes de África, Asia y el resto de América, donde se han evidenciado aportes nutricionales importantes, así como altos niveles de vitaminas y minerales, además de poseer la mayor parte de los aminoácidos esenciales, como se describe detalladamente mediante la Tabla 1. Mientras que en aspectos medicinales, el ñame se ha empleado para reducir los niveles de glucosa en la sangre, el mejoramiento de enfermedades cardiovasculares y en el aprovechamiento de sus propiedades antifúngicas (Qin et al., 2009), lo que indica que el consumo de este producto en ámbitos internacionales no solo representa beneficios nutricionales relevantes, sino que también posee ciertas propiedades fitoterapéuticas que contribuyen al bienestar de la salud física de los consumidores interesados en el consumo cotidiano de este tubérculo, convirtiéndolo en una alternativa de uso alimentario para la obtención de productos como el soufflé, los flanes, los bizcochos, las croquetas o el tradicional puré africano conocido como "fufu".

En este sentido, una alternativa de procesamiento para el ñame que puede contribuir a la problemática que afronta dicho producto, es la obtención de sus almidones, considerándose que las fuentes principales para la obtención de almidones naturales son los cereales, las raíces, los tubérculos y las leguminosas, siendo los almidones más utilizados los que se obtienen a partir de fuentes amiláceas como el maíz, la papa, la yuca y el arroz; sin embargo, existen otras fuentes naturales no convencionales que contienen almidones y que son menos conocidas, como lo es el ñame y en este caso especial, el que se cultiva en Colombia y específicamente en el departamento de Sucre, destacando las especies Criollo (Dioscorea alata), Espino (Dioscorea rotundata) y Diamante 22. 
Tabla 1: Información nutricional del ñame adaptada de Reina Aranza, (2012). ( ${ }^{\star}$ Cantidad por cada 100 gramos).

\begin{tabular}{|c|c|c|}
\hline Componente & Cantidad $^{*}$ & Unidad de medida \\
\hline Agua & 69,6 & Gramos \\
\hline Proteínas & 1,53 & Gramos \\
\hline Carbohidratos & 27,88 & Gramos \\
\hline Azúcar & 0,5 & Gramos \\
\hline Hierro & 0,54 & Miligramos \\
\hline Fosforo & 55 & Miligramos \\
\hline Sodio & 9 & Miligramos \\
\hline Calcio & 17 & Miligramos \\
\hline Magnesio & 21 & Miligramos \\
\hline Potasio & 816 & Miligramos \\
\hline Zinc & 0,24 & Miligramos \\
\hline Vitamina C & 17,1 & IU \\
\hline Vitamina A, IU & 138 & Miligramos \\
\hline Riboflavina & 0,032 & Miligramos \\
\hline Vitamina B-6 & 0,293 & Microgramos_RAE \\
\hline Vitamina A, RAE & 7 & Miligramos \\
\hline Vitamina E & 0,35 & Miligramos \\
\hline Colesterol & 0 & Gramos \\
\hline Grasa & 0,17 & \\
\hline
\end{tabular}

Es importante señalar que los almidones, suelen utilizarse para la elaboración de un amplio grupo de productos de repostería, como es el caso de la fabricación de galletas, panes, pudines, tortas entre otros. El papel de los almidones en estos productos figura fundamentalmente como estabilizante, emulsificante, espesante y adhesivo, con lo cual, se contribuye al mejoramiento de las propiedades texturales, por nombrar algunas funciones. Así mismo, se resalta que, al momento de la fabricación de estos productos, los almidones son sometidos a altas temperaturas durante los procedimientos de cocción, causando su gelatinización; con ello, los almidones gelatinizados se retrogradan, especialmente durante la ocurrencia de los procedimientos de enfriamiento y posterior almacenamiento, influyendo este fenómeno en la calidad final de aquellos productos que lo contengan dentro de su formulación (Biduski et al., 2018). Los almidones nativos obtenidos de las diferentes fuentes amiláceas, poseen propiedades específicas y comportamientos diferentes con relación a sus propiedades funcionales, lo cual, va a estar condicionado sin duda alguna por la procedencia de su fuente natural de obtención, además poseen propiedades específicas y se comportan de forma variable con relación a sus propiedades fisicoquímicas y tecno-funcionales, lo que depende de las características estructurales, tales como la proporción relativa de amilosa y amilopectina, distribución de longitud de cadena y frecuencia de ramificación de estos dos componentes, organización molecular, forma y tamaño de gránulo y contenido de lípidos y proteína. Todas estas características estructurales influyen en su comportamiento durante el procesamiento y son propias de cada tipo de almidón. Por tal motivo, se hace necesario estudiar y profundizar en las diversas fuentes no convencionales de almidones que actualmente existen, lo que podría resultar un aporte valioso para la generación de alternativas de mejoramiento y de aplicación en diferentes sectores económicos, principalmente para las industrias de alimentos, cosméticos, papel, textil y otras (Sívoli et al., 2012).

Las especies Espino y Criollo son las de mayor distribución e importancia económica en el mundo, como también son las de mayor área sembrada y las más demandadas en Colombia. Dioscorea alata es originaria del sudeste asiático y cuenta con las mejores características en cuanto a respuesta a la propagación, rendimiento, vigor y capacidad de almacenamiento; mientras que Dioscorea rotundata es originaria del occidente de África y aunque no iguala las propiedades de la especie Dioscorea alata, es menos susceptible a hongos fitopatógenos que causan la antracnosis. La especie Diamante 22 es un clon originario de Filipinas, tolerante a la antracnosis. Comparando las tres especies, los de mejor capacidad de almacenaje son el ñame Diamante 22 y el Criollo, mientras que el ñame Espino no se puede almacenar porque pierde peso rápidamente. Teniendo en cuenta que se ha demostrado que, para una misma región y una misma especie, es posible encontrar distintos contenidos de almidones que pueden ser clasificados en almidones tipo A, tipo B, tipo C, y que cuyas diferencias obedecen a las configuraciones obtenidas en los contenidos de amilosa y amilopectina de dichos almidones (He y Cunxu, 2017); así que como también de que estas configuraciones pueden estar relacionadas con cambios irreversibles en las propiedades del almidón como lo son el 
hinchamiento, formación de geles y disolución que son aspectos que fundamentalmente deben determinarse en el diseño y desarrollo de productos, fue necesario plantear como objetivo realizar un análisis de las curvas de gelatinización de los almidones nativos extraídos de las especies de ñame Criollo, Espino y Diamante 22, para comprender su comportamiento bajo diferentes escenarios de procesamiento.

\section{METODOLOGÍA}

Se describen a continuación los procedimientos experimentales y de análisis que se desarrollaron para la extracción y caracterización de los almidones nativos de las especies de ñame abordadas en este estudio.

\section{Obtención del almidón}

Para la extracción del almidón, se empleó un equipo de burbujeo contínuo a escala piloto ubicado en la planta de Operaciones Unitarias de la Universidad de Sucre (Sincelejo-Sucre, Colombia), el cual, operó en condiciones ambientales con una relación solución de muestra:agua de 1/8 y cuyo fundamento fue la flotación por la presencia de aire. El proceso consistió en las operaciones preliminares de adecuación de la materia prima, operación del equipo que duró de 40 min a 60 min y post-operación que consistió en la sedimentación y secado, el cual se llevó a cabo en un horno convectivo a una temperatura de operación de $40{ }^{\circ} \mathrm{C}$ por un periodo de tiempo de $24 \mathrm{~h}$, luego se realizó el proceso de molienda y tamizado utilizando una malla número 100 y almacenado en recipientes sellados herméticamente (Pérez et al., 2015).

\section{Apariencia microscópica}

Para el análisis de almidones, se utilizó un microscopio óptico de alta resolución M.O.A.R. (Nikon Microphot, Japón) con software Leica Qwin 550. Las muestras se observaron a 40x. Se utilizó la técnica de contraste diferencial de interferencia (DIC), dado que esta técnica aprovecha las diferencias en el índice de refracción y el espesor de la muestra, permitiendo determinar la topografía del objeto de estudio (Toshio et al., 2000)

\section{Composición proximal y $\mathrm{pH}$}

El análisis proximal de los almidones de ñame se realizó de acuerdo a los métodos oficiales descritos por la AOAC (2012), para determinar humedad (método 925.10), proteína bruta (método 920.87), grasa bruta (método 920.85), ceniza (método 923.03), fibra bruta (método 920.86) y pH (método 943.02).

\section{Determinación de amilosa y amilopectina}

La cantidad de amilosa fue determinada aplicando el método colorimétrico de yodo estandarizado, siguiendo la norma ISO 6647- 1:2007 (ISO, 2007). Se realizó una curva de calibración utilizando un estándar de amilosa de papa, adquirido de Sigma Aldrich (St Louis. MI USA). La absorbancia de las muestras se midió a $620 \mathrm{~nm}$ empleando un espectrofotómetro (Thermo Scientific, Evolution 60S, USA). Para calcular la cantidad de amilosa en las muestras, inicialmente se restó el valor del blanco al de la lectura de absorbancia de la muestra, seguidamente se restó la intercepción y finalmente se dividió la absorbancia corregida por la pendiente. El contenido de amilopectina, se calculó por diferencia al 100\% del contenido de amilosa mediante colorimetría (Morrison y Laignelet, 1983).

\section{Comportamiento de la pasta de almidón en frío y caliente (Curvas de gelatinización)}

Se determinó el comportamiento de la pasta en frío y caliente, mediante un test de gelificación guiados en la metodología propuesta por Figueroa et al., (2016), donde se realizaron curvas de gelatinización en un reómetro (Anton Paar, MCR 302, Austria) utilizando suspensiones de $2 \mathrm{~g}$ de muestra en base seca en $25 \mathrm{~mL}$ de agua destilada. La suspensión fue sometida a calentamiento, inicialmente a $50^{\circ} \mathrm{C}$ por un min, luego fue elevada a $95^{\circ} \mathrm{C}$ en $7,5 \mathrm{~min}$; con una rata de calentamiento de $6^{\circ} \mathrm{C}$ por minuto, se mantuvo a dicha temperatura por 5 min y posteriormente fue enfriada hasta $50^{\circ} \mathrm{C}$ en $7,5 \mathrm{~min}$. Finalmente, se mantuvo durante 2 min a temperatura de $50^{\circ} \mathrm{C}$. La velocidad del husillo (Anton Paar, ST24-2D/2V, Austria) fue de 960 r.p.m. durante los primeros $10 \mathrm{~s}$ permitiendo dispersar uniformemente la suspensión de almidón, y luego reducida a 160 r.p.m. para el resto del experimento. Los parámetros evaluados fueron la temperatura inicial de pastificación o gelificación, la viscosidad máxima, viscosidad a $95^{\circ} \mathrm{C}$ por $5 \mathrm{~min}$, viscosidad final, estabilidad (breakdown) y asentamiento (setback). Los resultados obtenidos del ensayo, fueron procesados mediante el software RheoCompass 1.12.

Análisis estadístico

Para el análisis estadístico de los datos obtenidos, se realizó un experimento bajo configuración de diseño completamente aleatorizado unifactorial en tres niveles (DCA), donde el factor de variación estuvo condicionado por los niveles de la muestra de la especie de ñame que se extrajo el almidón (almidón espino, almidón criollo y almidón diamante 22). Para establecer la significancia del efecto de la variable independiente 
sobre las variables dependientes, se aplicó un análisis de varianza (ANOVA) $(p \leq 0,05)$ complementado por un test de comparaciones de rangos múltiples de Tukey $(\alpha=0,05)$, para comparar los valores medios. Los experimentos se realizaron por triplicado para cada condición de tratamiento. Los datos experimentales fueron analizados estadísticamente utilizando el software estadístico R-Studio versión Versión 1,1,463 - ( ) 20092018 bajo licencia General Public License (GNU).

\section{RESULTADOS Y DISCUSIÓN}

Seguidamente, se presentan los resultados obtenidos del proceso de caracterización de los almidones nativos de las especies de ñame en estudio.

\section{Apariencia microscópica}

Las microfotografías de los gránulos de almidones nativos se muestran en la Figura 1, en donde se observan las formas típicas lenticulares y redondas, características de estos productos, cuyas observaciones son similares a la reportada en otra investigación (Hoover, 2002).

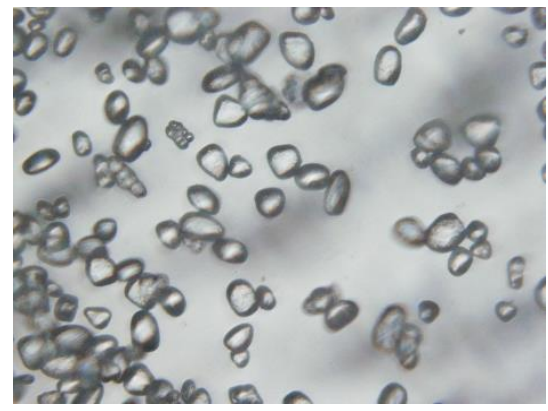

a.

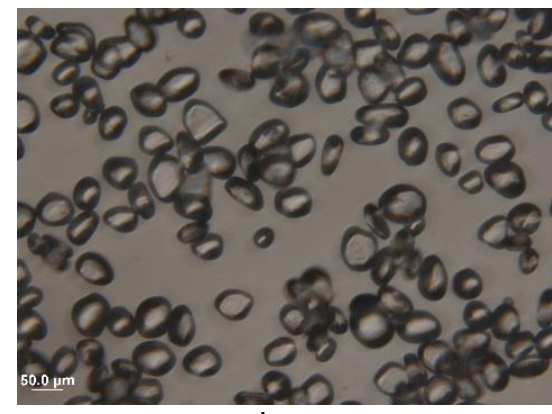

b.

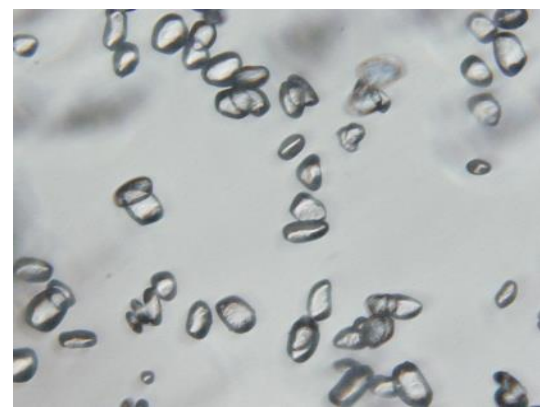

C.

Fig.1: Microfotografías 40x. Almidones nativos: a. Ñame Criollo, b. Ñame Diamante 22, c. Ñame Espino.

De igual forma, es posible observar en la micrografía referente a los almidones, que los microgránulos de los almidones nativos de estas especies presentan características físicas no uniformes con respecto a tamaño y forma en los gránulos que lo conforman, lo cual corresponde a la naturaleza de los almidones procedentes de órganos aéreos en las plantas (Meaño Correa et al., 2016). Adicionalmente, una característica correspondiente con el fenómeno de birrefringencia es un factor común en los almidones evaluados, por lo que se espera que estos almidones tengan la propiedad óptica de separar un rayo de luz que incida sobre ellos, polarizándolo de manera perpendicular en dos índices distintos de refracción, lo cual, es una propiedad física relevante para evaluar al momento de contemplar la aplicación de este tipo de almidones en matrices alimentarias fotosensibles. Con respecto a la aplicación de estos almidones para el sector no alimentario, es necesario considerar que de acuerdo a las características no uniformes de los diámetros de los gránulos es recomendable contemplar su uso para la elaboración de productos cosmetológicos por sus buenas propiedades higroscópicas, o como agentes con la capacidad de encapsular el color o las esencias como en el caso de las pinturas, barnices o bioplásticos (Kaur et al., 2002).

\section{Caracterización Fisicoquímica de almidones de ñame}

La composición fisicoquímica de los almidones de las especies de ñame Criollo, Espino y Diamante 22, se ilustran en la Tabla 2, en esta puede observarse que las especies presentan diferencias estadísticamente significativas $(\mathrm{p} \leq 0,05)$ en cuanto al contenido de humedad, contenido de proteína y para el $\mathrm{pH}$, lo cual significa que existen diferencias en los valores medios de estos componentes producto de las especies de ñame evaluadas. Con relación al contenido de ceniza, grasa y fibra los almidones extraídos de estas especies no presentan entre sí diferencias estadísticamente significativas, por tanto, las variaciones de las especies de ñame no representan valores medios en estos componentes que permitan diferenciar las proporciones de los mismos en el producto, lo cual se explica porque tanto el contenido de grasa y de fibra no son componentes mayoritarios de la fisiología composicional del tubérculo. Por su parte, se resalta la igualdad del contenido de materia seca en las muestras de los materiales, lo cual es coherente con la igualdad estadística en el contenido de cenizas.

El mayor contenido de humedad lo presenta el almidón de ñame Diamante 22, con diferencias estadísticamente significativas con respecto a los ñames Espino y Criollo que presentaron un menor contenido de este componente es su estructura composicional. El contenido de humedad de los almidones presenta un valor aceptable dentro de la escala de Moorthy, indicando que el contenido de humedad de los almidones varía entre $6 \%-16 \%$; contenidos superiores pueden conducir a daño microbiano y subsecuente deterioro de la calidad (Moorthy, 2002). También con estos valores es posible predecir que, a mayor contenido de 
humedad, se ocasiona un aumento en la temperatura de fusión de los cristales de almidón, lo cual puede verse reflejado en una mayor temperatura inicial de gelatinización, que este caso específico corresponde a la especie de ñame Criollo (Tabla 3) (Rodríguez et al., 2001).

De forma general, las especies Criollo y Espino coinciden en el contenido de humedad reportados por Alvis et al., (2008) en diferentes variedades de Dioscorea alata (8,660 \% a 10,22 \%), siendo inferiores a los reportados por Pérez et al., (2013) para cinco especies de Dioscorea (11,80 \% a 12,70 \%) en cuyo rango solo es posible ubicar al ñame Diamante 22.

Tabla 2: Composición fisicoquímica de la variedad de ñame Criollo, Espino y Diamante 22.

\begin{tabular}{|c|c|c|c|}
\hline Parámetro & Criollo & Espino & Diamante 22 \\
\hline Humedad (\%) & $7,570 \pm 0,9400^{\mathrm{b}}$ & $8,100 \pm 0,8300^{\mathrm{b}}$ & $12,11 \pm 0,8500^{\mathrm{a}}$ \\
\hline Ceniza (\%) & $0,4300 \pm 0,02000^{\mathrm{a}}$ & $0,4400 \pm 0,06000^{\mathrm{a}}$ & $0,5000 \pm 0,02000^{\mathrm{a}}$ \\
\hline Proteína (\%) & $1,410 \pm 0,05000^{\mathrm{a}}$ & $1,680 \pm 0,01000^{\mathrm{a}}$ & $0,9500 \pm 0,2500^{\mathrm{b}}$ \\
\hline Grasa (\%) & $0,3500 \pm 0,1400^{\mathrm{a}}$ & $0,3300 \pm 0,1500^{\mathrm{a}}$ & $0,1600 \pm 0,05000^{\mathrm{a}}$ \\
\hline Fibra (\%) & $0,01000 \pm 0,02000^{\mathrm{a}}$ & $0,02000 \pm 0,02000^{\mathrm{a}}$ & $0,02000 \pm 0,01000^{\mathrm{a}}$ \\
\hline pH & $6,180 \pm 0,06000^{\mathrm{b}}$ & $6,850 \pm 0,02000^{\mathrm{a}}$ & $6,200 \pm 0,05000^{\mathrm{b}}$ \\
\hline Amilopectina (\%) & $75,15 \pm 0,1500^{\mathrm{b}}$ & $76,40 \pm 0,8200^{\mathrm{a}}$ & $74,50 \pm 0,2100^{\mathrm{b}}$ \\
\hline Amilosa (\%) & $24,85 \pm 0,1500^{\mathrm{a}}$ & $23,64 \pm 0,8200^{\mathrm{b}}$ & $25,53 \pm 0,2100^{\mathrm{a}}$ \\
\hline
\end{tabular}

Los almidones obtenidos a partir de estas tres especies no presentaron diferencias estadísticas significativas $(p \leq 0,05)$ en el contenido de ceniza. Los valores obtenidos fueron superiores a los informados para especies silvestres de ñame (Dioscorea spp), Dioscorea alata y esculenta, cuyos valores oscilan entre 0,1300 \% y 0,3200 \% (Pérez et al., 2013; Jayakody et al., 2007). Este mayor contenido de cenizas puede estar relacionado posiblemente con un aumento en el contenido de minerales en los almidones de estas especies de ñame, ya que algunos estudios demuestran que el contenido de fósforo y de algunos minerales en ñame influyen en el contenido final de las cenizas (Hoover, 2002).

El mayor contenido de proteína en el almidón fue obtenido en la especie de ñame Espino (1,684 \%) presentando igualdad estadística con la especie Criollo y el menor valor lo presentó la especie de ñame Diamante 22. Se encontraron diferencias estadísticamente significativas $(p \leq 0,05)$ entre las especies Criollo Diamante 22 y Espino - Diamante 22. Los resultados obtenidos fueron superiores a los reportados para distintas especies de Dioscorea alata y para almidones nativo y modificados de la especie Dioscorea opposita cuyos porcentajes de proteína variaron entre 0,1000 \% y 0,4900 \% (Huang et al., 2016; Alvis et al., 2008). Los valores determinados de proteína se encuentran en un nivel superior al permitido por la FDA en almidones de maíz (0,3500 \%), lo cual los convierte en almidones no aptos para la elaboración de jarabes debido a su alto contenido de proteínas, ya que no se evitaría las reacciones de Maillard que se pueden presentar durante el proceso de producción de estos productos (Zajac, 1989). Por otro lado, estos valores son importantes al tener en cuenta la nutrición de los seres humanos, ya que constituye un producto ampliamente consumido por la población de menores recursos económicos y son una opción latente para la obtención de un contenido proteico colateral al consumo de carbohidratos aportados por este tubérculo.

Los almidones no presentaron diferencias significativas $(p \leq 0,05)$ en el contenido de grasa, presentando valores superiores a los reportados por Alvis et al., (2008) para cuatro especies $(0,0000 \%$ a 0,0600 \%), pero que están acordes a los informados por Pacheco y Techeira, (2009), en donde el contenido ideal de lípidos debe ser menor al 0,5\%, lo cual favorece las propiedades de hinchamiento y solubilización debido a que se evita la formación de un complejo con la amilosa, que reprime estas propiedades y por el cual se requeriría de temperaturas superiores a los $125^{\circ} \mathrm{C}$ para que se puedan dar las propiedades en mención. En este sentido, se ha demostrado que el valor nutricional de los almidones es altamente energético, además de ser una gran fuente de carbohidratos, sin embargo, presentan bajos contenidos de proteína y grasa, por lo que podrían utilizarse para mejorar el contenido energético de algunos alimentos, sin suministrar grasa adicional, por lo que también pueden ser un alimento acorde a las actuales tendencias alimentarias de consumo de productos bajos en grasa (Vargas Aguilar y Hernández Villalobos, 2013). Por otro lado, se detectaron bajos contenidos de fibra, sin diferencias estadísticas significativas entre estas especies. De igual forma, el pH determinado en el almidón Espino fue estadísticamente diferente $(p \leq 0,05)$ al almidón de la especie alata. Los resultados obtenidos estuvieron dentro del rango reportado por Techeira (2014) para almidones y harina de ñame de la especie Dioscorea alata $(5,910$ a 7,310$)$. 
En cuanto al contenido de amilosa, en la Tabla 2 se observa que, el mayor contendido de este material se encuentra en la especie Diamante 22 (25,53 \%), mientras que el menor contenido de amilosa se encuentra en la especie Espino (23,64\%), obteniendo diferencias estadísticamente significativas con respecto a las otras especies evaluadas. También, se evidencia un mayor contenido de amilopectina para la especie Espino y el menor contenido para la especie Diamante 22. Asimismo, se evidenciaron diferencias significativas entre estas dos especies. Los resultados obtenidos de amilosa se encontraron dentro del rango reportado para Dioscorea esculenta y alata (19,98\% a 29,29 \%) (Jayakody et al., 2007) y para almidón nativo y modificado enzimáticamente de maíz (23,47 \% a 28,95 \%) (Benavent y Rosell, 2017). Al realizar una comparación de los porcentajes de amilosa que presenta el almidón de las especies en estudio, con otros productos que se cultivan en Colombia, como son, la papa, el maíz y el trigo, que de forma respectiva presentan los siguientes contenidos de amilosa $20 \%, 25 \%$ y $25 \%$, y que por ello, se han considerado como almidones formadores de geles, podemos ubicar los obtenidos en esta investigación dentro de esta misma denominación (Pacheco y Techeira, 2009).

Por otro lado, los valores de amilopectina son superiores a los infor mados para Dioscorea bulbifera (70,62 \%) (Araujo et al., 2004), Disocorea Trifida en sus variedades morado (56,67 \%) y blanco (65,28 \%), así como también en Dioscorea bulbifera $L(69,37 \%)$, lo cual puede conducir a la producción de geles más elásticos al ser comparados con esta última especie (Zhou et al., 1998). Estos distintos contenidos de amilosa y amilopectina han sido atribuidos principalmente a la variación de la fuente de obtención de almidón, clima, edad y sitio de cultivo (Onitilo et al., 2007; Vargas Aguilar y Hernández Villalobos, 2013).

\section{Caracterización de la viscosidad de almidones de ñame}

En la Tabla 3 y Figura 2, se muestran los resultados obtenidos al analizar las curvas de gelatinización de los almidones, se evidenciaron diferencias estadísticas significativas $(p \leq 0,05)$ entre la especie Espino con respecto a Criollo y Diamante 22 , entre los cuales no se evidenciaron diferencias estadísticas $(p \leq 0,05)$.

Tabla 3: Características viscográficas de almidones.

\begin{tabular}{|l|c|c|c|}
\hline \multicolumn{2}{|c|}{ Características } & \multicolumn{3}{c|}{ Especie } \\
\cline { 2 - 4 } & Espino & Criollo & Diamante 22 \\
\hline Temperatura inicial gelatinización $\left({ }^{\circ} \mathrm{C}\right)(\mathrm{A})$ & $80,43 \pm 0,3512$ & $84,03 \pm 0,3055$ & $82,30 \pm 0,8718$ \\
\hline Viscosidad máxima (B) (Pa.s) & $2,752 \pm 0,1579$ & $1,367 \pm 0,0730$ & $1,968 \pm 0,4354$ \\
\hline Viscosidad a $95^{\circ} \mathrm{C}(\mathrm{C})(\mathrm{Pa} \cdot \mathrm{s})$ & $2,476 \pm 0,1298$ & $1,344 \pm 0,1075$ & $1,956 \pm 0,4805$ \\
\hline Viscosidad a $95^{\circ} \mathrm{C}, 5 \mathrm{~min}(\mathrm{D})(\mathrm{Pa} \cdot \mathrm{s})$ & $2,259 \pm 0,2014$ & $1,296 \pm 0,0831$ & $1,868 \pm 0,4171$ \\
\hline Viscosidad a $50^{\circ} \mathrm{C}(\mathrm{E})(\mathrm{Pa} \cdot \mathrm{s})$ & $4,169 \pm 0,5799$ & $2,100 \pm 0,1661$ & $3,232 \pm 0,6098$ \\
\hline Viscosidad final $50{ }^{\circ} \mathrm{C}(\mathrm{F})(\mathrm{Pa} \cdot \mathrm{s})$ & $4,226 \pm 0,5779$ & $2,161 \pm 0,1897$ & $3,331 \pm 0,5970$ \\
\hline Breakdown (B-D) (Pa:s) & $0,4930 \pm 0,0869$ & $0,07066 \pm 0,0106$ & $0,1000 \pm 0,0035$ \\
\hline Setback (F-D) (Pa.s) & $1,967 \pm 0,4136$ & $0,865 \pm 0,0027$ & $1,463 \pm 0,0032$ \\
\hline
\end{tabular}

El almidón en presencia de agua y con el suministro adecuado de energía sufre un proceso de gelatinización en el cual se rompe su estructura cristalina pasando de gránulos insolubles hasta la obtención de una solución de sus moléculas, originando pastas viscosas. Las moléculas de amilosa durante este proceso se difunden en el agua formando un gel, mientras que la amilopectina pierde su orden cristalino (Singh et al., 2001). Esta transición de orden-desorden que sufren los polímeros de almidón al ser sometidos a calentamiento son las que impactan en el procesamiento, calidad y estabilidad de los productos basados en almidón. Durante el proceso de gelatinización, el orden molecular dentro de los gránulos es destruido gradual e irreversiblemente, por esto la temperatura de gelatinización es característica para cada tipo de almidón y depende fundamentalmente de la transición vítrea de la fracción amorfa del almidón. La temperatura de gelatinización se convierte en un índice del orden intragranular del almidón, lo que quiere decir que a mayor temperatura de gelatinización, mayor es el grado de ordenamiento entre las macromoléculas presentes al interior del gránulo de almidón, los resultados obtenidos permiten inferir que la estructura de almidón con mayor orden intragranular se obtiene de la especie de ñame Criollo que presenta una temperatura de gelatinización de $84,03^{\circ} \mathrm{C}$, seguida en orden descendente de las especies de ñame Diamante 22 y Espino con valores respectivos de $82,30^{\circ} \mathrm{C}$ y $80,43^{\circ} \mathrm{C}$, lo que puede indicar que el almidón presente en esta última especie iniciaría el proceso de absorción de agua, hinchamiento y gelificación a una menor temperatura y necesitaría de un menor tiempo de cocción. Algunos factores asociados con estas diferencias han sido atribuidos a la relación amilosa/amilopectina, $\mathrm{pH}$, origen botánico del almidón o tamaño del gránulo. 
En la etapa de calentamiento la viscosidad aumentó gradualmente hasta un valor máximo y también en todos los casos se observó que la viscosidad aumentó durante la etapa de enfriamiento, hecho que puede atribuirse a la reorganización de las cadenas lineales durante la etapa de calentamiento, y un aumento del número de zonas de unión durante la formación de la pasta y, por tanto, una red que retiene más agua (Mali et al., 2003).La viscosidad máxima se obtuvo en almidón de ñame Espino (2,752 Pa.s), seguido en orden descendente por los valores obtenidos para almidón de ñame Diamante 22 y Criollo (1,968 Pa.s y 1,367 Pa.s), al realizar una comparación entre estas especies, los datos obtenidos pueden indicar que la suspensión de almidón de ñame Criollo no es de fácil cocción, lo cual es consistente con los planteamientos realizados en el párrafo anterior.

La mayor viscosidad a $50{ }^{\circ} \mathrm{C}$ la presenta el almidón obtenido de la especie de ñame Espino con un valor de 4,226 Pas; que se puede atribuir aun probable reordenamiento lineal de las moléculas de amilosa. $\mathrm{A} 95^{\circ} \mathrm{C}$ por un periodo de 5 min las viscosidades de los almidones de todas las especies en estudio mostraron una disminución con respecto a los valores obtenidos de viscosidad máxima, siendo estos valores 2,259 Pa.S, 1,868 Pas, y 1,296 Pas, que en el orden respectivo corresponden a las especies de ñame Espino, Diamante 22 y Criollo. Este fenómeno podría ser posible debido a una orientación de las moléculas de almidón que son solubles, hacia la misma dirección en la cual es agitado el sistema, fenómeno conocido como "Shear Thining" (Hoseney, 1986).

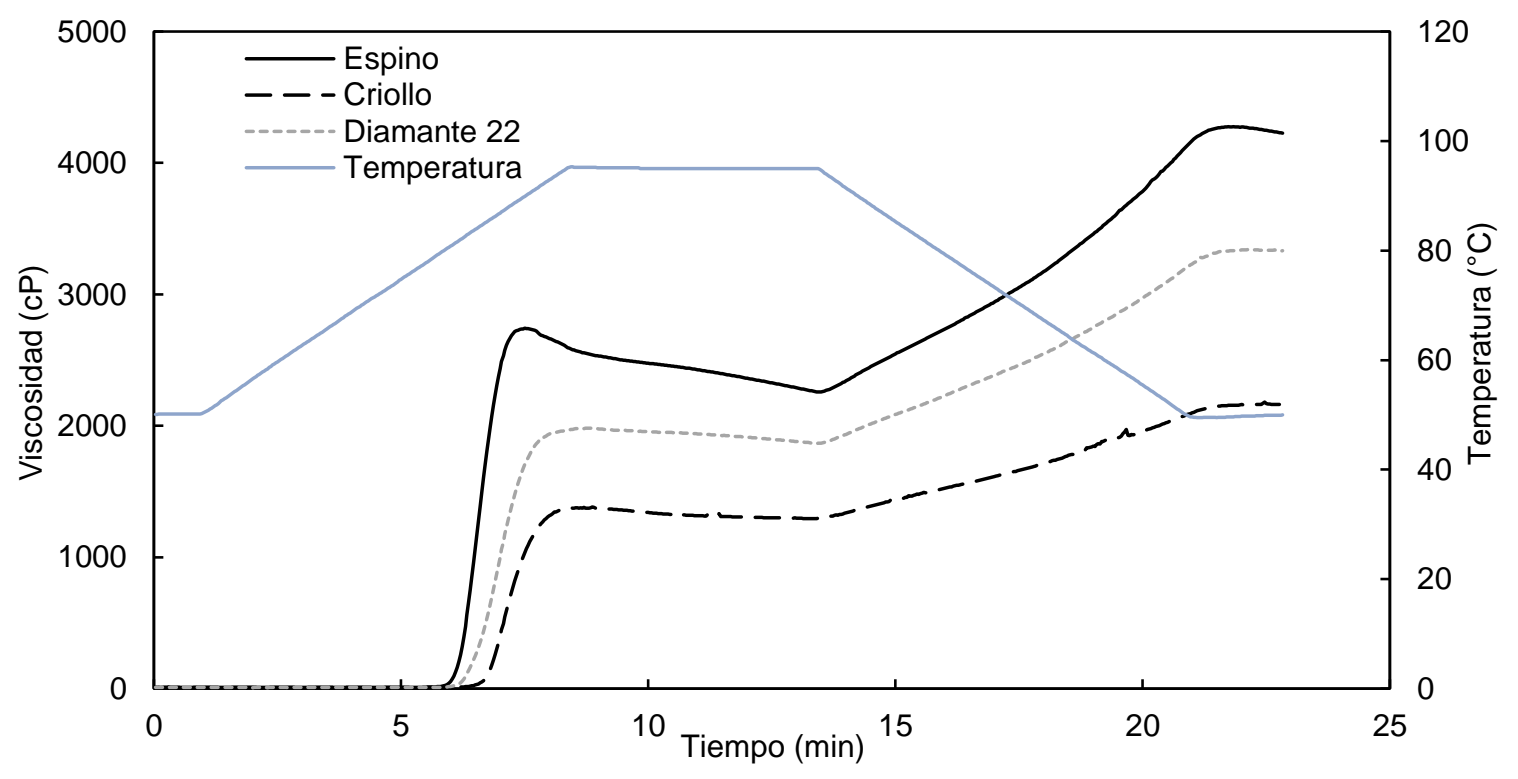

Fig.2: Viscoamilograma de almidones.

Por otro lado, se encontró que la especie Espino, presentó mayor viscosidad inicial y pico de viscosidad, pero en la etapa de calentamiento constante mostró una disminución, lo que se contrasta con el mayor valor de estabilidad, reflejado en una mayor capacidad de hinchamiento, menor estabilidad en la cocción y que puede estar relacionado con una disminución de la viscosidad una vez alcanzado el valor máximo; mientras que los almidones obtenidos de la especie Dioscorea alata, aunque presentaron menores viscosidades revelaron un comportamiento en caliente más estable evidenciado en sus menores valores de breakdown, comportamiento que puede ser debido a una mayor tendencia del almidón de ñame Espino a retener agua en el proceso de calentamiento (Belei et al., 2006). Asimismo, los almidones obtenidos de la especie Dioscorea alata presentaron una menor tendencia a la retrogradación, reflejados en bajos valores de setback, producto de la disminución en la viscosidad de las suspensiones durante el enfriamiento. Resultados similares fueron informados por Paternina et al., (2016), al realizar estudios de especies similares y compararlas con almidones nativos de otros productos.

\section{CONCLUSIONES}

De los resultados y análisis realizados es posible deducir que el almidón obtenido del ñame Espino puede ser considerado como un buen agente espesante, debido a la mayor viscosidad desarrollada durante las fases de calentamiento y enfriamiento. Los gránulos de ñame Criollo y Diamante 22 presentaron una mayor estabilidad y menor tendencia a la retrogradación, potencializando su uso en alimentos que requieran mantener un gel de consistencia estable a procesos de cocción y en productos que necesiten enfriamiento durante su preparación, lo que sugiere su posible uso en formulaciones de alimentos en donde se requiere el desarrollo de una rápida viscosidad y un gel de consistencia estable. 


\section{AGRADECIMIENTOS}

Los autores agradecen el apoyo de la Corporación Universitaria del Caribe (CECAR) a través de la financiación del proyecto de convocatoria interna VICET +l 0402016.

\section{REFERENCIAS}

Alvis, A., C. Vélez, H. Villada y M. Rada-Mendoza, Analisis fisico-químico y morfológico de almidones en ñame, yuca, papa y determinación de la viscosidad de pastas, Información Tecnológica, 19(1), 19-28 (2008)

Alvis, A., J. Rodríguez y G. Arrázola, Efecto del freído sobre cambios de humedad y absorción de aceite en ñame (Dioscorea rotundata P), Agronomía Colombiana, 34(1Supl), S314-S316 (2016)

AOAC International, Official Methods of Analysis of AOAC International (19a Ed.). MD, USA.: Editorial Gaithersburg (2002)

Araujo, C., A. Rincón y F. Padilla, Caracterización del almidón nativo de Dioscorea bulbifera L, Archivos Latinoamericanos de Nutrición, 54(2), 241-245 (2004)

Beleia, A., S. Butarelo y R. Silva, Modeling of starch gelatinization during cooking of cassava (Manihot esculenta Crantz), Science and Technology, 39(4), 400-405 (2006)

Benavent, Y. y C. Rosell, Comparison of porous starches obtained from different enzyme types and levels, Carbohydrate Polymers, 157, 533-540 (2017)

Biduski, B., W.M. Ferreria da Silva y otros cinco autores, Starch hydrogels: The influence of the amylose content and gelatinization method, International Journal of Biological Macromolecules, 113, 443-449 (2018)

DANE, Estadísticas Agroforestales 1987-2016 Ñame y Otros cultivos, Bogota (2016)

Figueroa, J., J. Salcedo y M. Rodríguez, Acetilación de almidón nativo de batata (Ipomeas batata L), Vitae, 23(1), S174S179 (2016)

González Vega, M. E., El ñame (Dioscorea spp.). Características, usos, Cultivos Tropicales, 33(4), 5-15 (2012)

He, W. y W. Cunxu, Progress in C-type starches from different plant sources, Food Hydrocolloids, 73, $162-175$ (2017)

Hoover, R., Effect of heat-moisture treatment on the structure and physicochemical properties of tuber and root starches, Carbohydrate Polymers, 49(4), 425-437 (2002)

Hoseney, R. C., Principles of Cereal Science and Technology, Minnesota, USA, American Association of Cereal Chemists (1986)

Huang, H., Q. Jiang y otros cinco autores, Preparation, physico-chemical characterization and biological activities of two modified starches from yam (Dioscorea Opposita Thunb.). Food Hydrocolloids, 55, 244-253 (2016)

ISO 6647-1: Rice- Determination of amylose content. Part one: Reference method, Geneva (2007)

Jayakody, L., R. Hoover, Q. Liu y E. Donner, Studies on tuber starches. II. Molecular structure, composition and physicochemical properties of yam (Dioscorea sp.) starches grown in Sri Lanka, Carbohydrate Polymers, 69(1), 148$163(2007)$

Kaur, L., N. Singh y N. S. Sodhi, Some properties of potatoes and their starches II. Morphological, thermal and rheological properties of starches, Food chemistry, 79(2), 183-192 (2002)

Mali, S., C. Ferrero y otros cuatro autores, Influence of $\mathrm{pH}$ and hydrocolloids addition on yam (Dioscorea alata) starch pastes stability. LWT-Food Science and Technology, 36(5), 475-481 (2003)

Meaño Correa, N., A. Ciarfella Pérez y A. Dorta Villegas, Caracterización morfológica y perfil viscoamilográfico del almidón nativo de ñame Congo (Dioscorea bulbifera L.), Saber, 28(2), 250-256 (2016)

Moorthy, S., Physicochemical and functional properties of tropical tuber starches: a review, Starch, 54(12), 559-592 (2002)

Morrison, W. R. y B. Laignelet, An improved colorimetric procedure for determining apparent and total amylose in cereal and other Starches, Journal of Cereal Science, 1, 19-35 (1983)

Onitilo, M. O., L.O. Sanni, O.B. Oyewole y B. Maziya-Dixon, Physicochemical and functional properties of sour starches from different cassava varieties, International Journal of Food Properties, 10(3), 607 - 620 (2007)

Pacheco, E. y N. Techeira, Propiedades químicas y funcionales del almidón nativo y modificado de ñame (Dioscorea alata), Interciencia, 34(4), 280-285 (2009)

Paternina, A., J. Figueroa, J. Salcedo y M. Cervera, Propiedades de empastamiento en almidones nativos de yuca, ñame y batata, Agronomía Colombiana, Suplemento 1(1), S402-S40 (2016)

Pérez, E., A. Rolland-Sabaté y otros cinco autores, Isolated starches from yams (Dioscorea sp) grown at the Venezuelan Amazons: Structure and functional properties, Carbohydrate Polymers, 98(1), 650-658 (2013)

Pérez, J., A. Fernández y J. Salcedo, Evaluación de un proceso continuo de burbujeo para recuperación de almidón y mucilago de ñame, Temas Agrarios, 20(1), 71-82 (2015) 
Qin, Y., X. Wu y otros cinco autores, Acute toxicity and sub-chronic toxicity of steroidal saponins from Dioscorea zingiberensis CH Wright in rodents, Journal of Ethnopharmacology, 126(3), 543-550 (2009)

Reina, Y., El cultivo del ñame en Colombia, Documentos de Trabajo sobre Economía Regional, Centro de Estudios Regionales, CEER, 12(168), 2-2 (2012)

Rodríguez, P., M.E. San Martín y G. González de la Cruz, Calorimetría diferencial de barrido y rayos-x del almidón obtenido por nixtamalización fraccionada, Superficies y Vacío. Soc. Mex. de Cien. Superficies y Vacío, 13, 61-65 (2001)

Singh, S., J. Singh y otros tres autores, Morphological, thermal and rheological properties of starches from different botanical sources. A review, Food Chemistry, 81, 219-231 (2001)

Sívoli, L., E. Pérez y P. Rodríguez, Structural analysis of the cassava native starch (Manihot esculenta C.) using morphometric, chemical, thermal and rheological techniques, Revista de la Facultad de Agronomía de la Universidad del Zulia, 29, 292-313 (2012)

Techeira, N., L. Sívoli y otro tres autores, Caracterización físicoquímica, funcional y nutricional de harinas crudas obtenidas a partir de diferentes variedades de yuca (Manihot esculenta Crantz), batata (Ipomoea batatas Lam) y ñame (Dioscorea alata), cultivada en Venezuela, Interciencia, 39(3), 191-197 (2014)

Toshio, O., Y. Tomoyuki, H. Shouji y M Takaakl, High-resolution Imaging of Starch Granule Structure using Atomic Force Microscopy, Starch/Stärke, 52(5), 150-153 (2000)

Vargas Aguilar, P. y D. Hernández Villalobos, Harinas y almidones de yuca, ñame, camote y ñampí: propiedades funcionales y posibles aplicaciones en la industria alimentaria, Tecnología en Marcha, 25(6), 37 - 45 (2013)

ZAJAC, P., Almidón de maíz, obtención y utilización, Cuba, Editorial Científico, Técnica, $92-113$ (1989)

Zhou, M., K. Robards, M. Glennie-Holmes y S. Helliwell, Structure and pasting properties of oat starch, Cereal Chemistry, 75(3), 273-281 (1998) 